\title{
Brodalumab in the treatment of psoriatic arthritis - the latest reports
}

\author{
Katarzyna Banaszczyk ID \\ Faculty of Medicine, Ludwik Rydygier Collegium Medicum in Bydgoszcz, Nicolaus Copernicus University in Torun, Bydgoszcz, Poland
}

\section{Dear Editor}

The quest to find an optimal and effective treatment for psoriatic arthritis (PSA) is expanding research to new molecules and inhibiting further pathways in the pathogenesis of inflammation in PsA.

In this report, I would like to draw your attention to brodalumab, a fully human IgG2 monoclonal antibody, which binds to human interleukin 17RA. Binding of this interleukin leads to blockade of the biological activity of the pro-inflammatory cytokines IL-17A, IL-17F, the heterodimer IL-17A/F, IL-17C and IL-17E [1]. It is worth being aware that IL-17A, IL-17F and the IL-17A/F heterodimer have a multidirectional effect; they induce pro-inflammatory mediators such as IL-6, GRO $\alpha$ and G-CSF from epithelial cells and fibroblasts, which affect the ongoing state of inflammatory tissues [2]. Brodalumab is used to treat moderate to severe plaque psoriasis (USA, Canada). Currently, brodalumab is also used to treat PsA but only in Japan [3].

It is worth emphasizing that brodalumab was effective in phase II clinical trials in PsA patients. Brodalumab has been used in a randomized, double-blind, and placebo-controlled trial. In this clinical trial, doses of $140 \mathrm{mg}$ and $280 \mathrm{mg}$ of brodalumab were administered once weekly for 12 weeks. Administration of brodalumab was associated with a significantly better clinical response compared to placebo (American College of Rheumatology 20 [ACR20] as the primary endpoint) [4]. The encouraging results of the second phase trials have prompted clinicians to conduct larger clinical trials. It is worth getting acquainted with the latest phase III study, the results of which were published at the end of October 2020.

What are the results of phase III trials of brodalumab in PsA? AMVISION-1 and AMVISION-2 trials

At the end of October 2020, the results of phase III clinical trials on the use of brodalumab in PsA were published [5]. These were double-blind, randomized, and placebo-controlled studies. The studies involved adult patients with active PsA who had been ill for at least 6 months. These patients did not tolerate traditional treatment or the treatment was insufficient. Additional inclusion criteria were having at least three painful and three swollen joints as well as active psoriatic lesions on the skin.

Patients (both in AMVISION-1 and AMVISION-2) were divided into three groups in a $1: 1: 1$ ratio and received subcutaneous brodalumab $140 \mathrm{mg}$, brodalumab $210 \mathrm{mg}$ and placebo, respectively [5]. This intervention was performed at week 0 and week 1 , and then every two weeks until week 24 . It should be noted that the primary endpoint was achievement of ACR20 at week 16 of treatment.

At week 16, it was noted that the primary endpoint was achieved by $45.8 \%$ of patients in the brodalumab $140 \mathrm{mg}$ group, $47.9 \%$ of patients in the brodalumab $210 \mathrm{mg}$ group and $20.9 \%$ of patients in the placebo group. It should be noted that similar results were noted at week 24 of this study. Patients receiving brodalumab achieved a greater percentage of ACR 50/70 compared to placebo. Moreover, patients receiving brodalumab had greater improvements in symptoms such as dactylitis and enthesitis. The study summary demonstrated that brodalumab had a good safety profile and the rate of serious adverse events was low [5].

\section{Brodalumab compared with other biologic thera- pies for psoriasis}

In the context of the effectiveness of brodalumab in PsA treatment, it is worth analyzing the previous studies on the efficacy of brodalumab in comparison to other biological therapies used in the treatment of moderate to severe psoriasis. The effectiveness of individual biological drugs was compared based on the results in the PASI scale (Psoriasis Area and Severity Index).

The most effective preparations turned out to be brodalumab and ixekizumab. Brodalumab was used at a dose of $210 \mathrm{mg}$ every two weeks, and ixekizumab 
at a dose of $80 \mathrm{mg}$, also every two weeks. Brodalumab at a dose of 210 mg was significantly more effective than drugs such as adalimumab (40 mg every two weeks), apremilast (30 mg twice daily), brodalumab $140 \mathrm{mg}$ administered every 2 weeks, etanercept (50 mg weekly), infliximab (5 mg/kg), secukinumab (300 mg) and ustekinumab (45 $\mathrm{mg}$ or $90 \mathrm{mg}$ - the dose depended on body weight). Brodalumab was more effective in PASI 100, 90, 75 and 50 scores. Studies have shown that $210 \mathrm{mg}$ brodalumab administered every two weeks is more effective in treating moderate to severe psoriasis than other typical biological therapies $[6,7]$.

Moreover, comparative studies are needed regarding the efficacy of brodalumab and other biological therapies in treating PsA.

In conclusion, brodalumab is already used successfully in plaque psoriasis, making it a good therapeutic option for patients with both skin and joint symptoms [8]. Its action is therefore multidirectional - it improves the clinical condition of the skin, joints and tendon attachments. Certainly, there will be more clinical trials with brodalumab in the future, as it is a promising therapeutic option for patients with psoriatic arthritis. It is worth monitoring the progress of clinical trials on brodalumab, as this drug offers hope for effective treatment of both psoriasis and psoriatic arthritis. Brodalumab, as a multi-directional drug, can find a place in dermatological and rheumatological therapy.

The author declares no conflict of interest.

\section{References}

1. EMA. Kyntheum summary of product characteristics. Available: https://www.ema.europa.eu/documents/product-information/kyntheum-epar-product-information_en.pdf [Accessed 21.02.2021].

2. Papp KA, Reid C, Foley P, et al. Anti-IL-17 receptor antibody AMG 827 leads to rapid clinical response in subjects with moderate to severe psoriasis: results from a phase I, randomized, placebo-controlled trial. J Invest Dermatol 2012; 132: 2466-2469, DOI: 10.1038/jid.2012.163.

3. Kyowa Kirin. LUMICEF $^{\oplus}$ Approved in Japan, 2016. Available: https://www.kyowakirin. co m/media_center/news_releases/ 2016/e20160704_01.html.

4. Mease PJ, Genovese MC, Greenwald MW, et al. Brodalumab, an anti-IL17RA monoclonal antibody, in psoriatic arthritis. N Engl J Med 2014; 370: 2295-2306, DOI: 10.1056/NEJMoa1315231.

5. Mease PJ, Helliwell PS, Hjuler KF, et al. Brodalumab in psoriatic arthritis: results from the randomised phase III AMVISION-1 and AMVISION-2 trials. Ann Rheum Dis 2021; 80: 185-193, DOI: 10.1136/annrheumdis-2019-216835.

6. Sawyer L, Fotheringham I, Wright E, et al. The comparative efficacy of brodalumab in patients with moderate-to-severe psoriasis: a systematic literature review and network metaanalysis. J Dermatolog Treat 2018; 29: 557-568, DOI: 10.1080/ 09546634.2018.1427205.

7. Kamata M, Tada Y. Efficacy and Safety of Biologics for Psoriasis and Psoriatic Arthritis and Their Impact on Comorbidities: A Literature Review. Int J Mol Sci 2020; 21: 1690, DOI: 10.3390/ ijms21051690.

8. Papp KA, Reich K, Paul C, et al. A prospective phase III, randomized, double-blind, placebo-controlled study of brodalumab in patients with moderate-to-severe plaque psoriasis. $\mathrm{Br} J$ Dermatol 2016; 175: 273-286, DOI: 10.1111/bjd.14493. 